\title{
El doble exilio de José Viñals
}

\author{
Benito Del Pliego y Andrés Fisher \\ Appalachian State University \\ Department of Foreign Languages \& Literatures \\ delpliegob@appstate.edu y fisheras@appstate.edu
}

\section{RESUMEN}

El exilio, desde sus definiciones más habituales, se refiere a la separación de una persona de la tierra en que vive, generalmente por motivos políticos. Desarrollos posteriores han ampliado el término, extendiéndolo más allá del desplazamiento geográfico más o menos forzoso. Se genera entonces la categoría del exiliado interno u outsider, aplicada a aquel que, crítico con su sociedad, se encuentra excluido de sus privilegios y poderes. Ambas categorías pueden aplicarse al poeta hispano-argentino José Viñals (Córdoba, Argentina, 1930-Málaga, 2009). Hijo de emigrantes españoles recientes, especialmente su padre, que había llegado de España bajo la dictadura de Primo de Rivera a Argentina pocos años antes de su nacimiento. Viñals repite este desplazamiento en sentido inverso en 1979, para escapar del peligro que suponía para él la dictadura militar de Videla, instalándose en España hasta su muerte. La situación de la poesía española que encuentra a su llegada, con una escena dominada por los discursos normalizadores que en un giro sorprendente se impusieron desde la Transición, supondrá la otra vertiente del exilio. La poesía que se legitima, conservadora en la expresión, anclada en la referencialidad y beligerante con los logros artísticos de la modernidad, hacen que su obra, junto con la de otros poetas notables ajenos a esa estética, experimente una situación periférica y de atención intermitente.

Palabras clave: José Viñals, inmigración latinoamericana, poesía contemporánea, España.

The double exile of José Viñals

\begin{abstract}
Exile, since its more usual definitions, refers to the state of being barred from the country in which a person normally lives, generally due to political reasons. Later developments had widened the term beyond the more or less forced geographical displacement. Thus, the category of the internal exiled or outsider came to existence, applied to a person who is excluded from the privileges and powers of his society because of his critical attitude. Both categories could be applied to the Spanish-Argentinean poet José Viñals (Córdoba, Argentina, 1930-Málaga, 2009), the son of recent Spanish emigrants particularly his father, arrived to


Argentina from Spain under Primo de Rivera's dictatorship few years before his birth. Viñals repeated this journey in the opposite direction in 1979, attempting to avoid the hazards of Videla's military dictatorship and getting settled in Spain ever since until his death. The situation of Spanish poetry he found at his arrival, with its scene dominated by approaches that proposed a normalized poetical expression in a surprising twist produced after the transition to democracy, meant for him the other side of exile. The poetry then legitimated, conservative in its expression, anchored in referentiality and contentious towards modernity's artistic accomplishments, relegated his and other remarkable poets' work alien to that approach, to a peripheral situation of sporadic critical attantion.

Key words: José Viñals, Latin-American immigration, contemporary poetry, Spain.

En su vigésimo segunda edición, el diccionario de la Real Academia Española define exilio, en sus dos primeras acepciones, como "Separación de una persona de la tierra en que vive. Expatriación, generalmente por motivos políticos". Es esta una definición con la que no resulta difícil estar de acuerdo, a la vez que puede parecer demasiado general para un mundo complejo como el actual, en el que los desplazamientos individuales y masivos se han incrementado de forma notable convirtiéndose en un signo de los tiempos. Así, diferentes teóricos han ampliado las definiciones del exilio llevándolo incluso a situaciones en las que el desplazamiento físico deja de ser un elemento imprescindible. Edward Said estudia el caso del intelectual en el exilio y las repercusiones de éste en la manera de mirar. En esa mirada que al producirse desde otro lugar se vuelve otra. Pero Said hace posible esta condición incluso para aquellos que no se han desplazado, "aquellos individuos disconformes con su sociedad y por tanto, outsiders y exiliados en lo que a privilegios, poder y honores se refiere" (Said 1996: 52)1.

Ambas vertientes pueden aplicarse a la peripecia vital y poética de José Viñals. La del que se desplaza y escribe desde otro lugar, pero también la de quien, que desde un espacio que deviene el suyo, lo hace desde la situación periférica que su inserción y el linaje del que se ha hecho parte determinan. La primera, la del desplazamiento físico, le precede y es parte suya desde incluso antes de su nacimiento, el que de cierta manera determina, para convertirse luego en una de sus señas vitales. La segunda, la de una posición excéntrica en un lugar al que ha hecho suyo, refleja la situación en que la entidad de su poética le ubicó en la escena de la poesía española que encontró al llegar a la península y que se mantuvo en las décadas siguientes.

José Viñals nació en 1930 en Corralito, pequeño pueblo de la provincia de Córdoba, en la Argentina rural. Sus padres eran españoles, emigrantes recientes, ella de Extremadura y especialmente él, de Cataluña, que había llegado pocos años antes del nacimiento del poeta, proveniente de una España que se encontraba bajo la dictadura de Primo de Rivera y que se aproximaba a una tragedia que tuvo hondas repercusiones en todos sus ámbitos generando un exilio cuya huella aun persiste.

\footnotetext{
1 La traducción es nuestra.
} 
La temprana muerte de su padre, panadero, cuando el poeta tenía apenas tres años de edad inaugura la sucesión de viajes y desplazamientos que, de mayor o menor extensión, se transformarán en una constante de su vida. Este primer viaje es breve y es al campo, a la chacra de los abuelos donde literalmente tiene una infancia a caballo, pues este era el medio de transporte que el niño Viñals usaba para asistir a la escuela. Pero el caballo fue mucho más que eso, transformándose en una presencia constante en su poesía. Caballo que entre muchas otras direcciones, puede metaforizar en el sentido del desplazamiento. Algo de lo que vemos en el poema que abre su libro Elogio de la miniatura.

Monté a mi caballo y me fui a recorrer el mundo. Por el ventanuco de una casa de adobe vi a una parturienta. En la cuneta vi una guitarra rota. A la entrada del bosque vi a una pequeña bruja -o lo que fuera- orinando sobre una rana viva. Ya no tengo tiempo de volver a mi país: soy demasiado viejo. ¿Qué haré con mis visiones? (Viñals 2002a: 9)

Desplazamiento que, una vez acabada la etapa escolar, lo lleva desde el campo a Córdoba capital donde se casa a temprana edad con quien será la compañera de toda la vida. Donde también se encuentra con Juan Larrea, y se hace militante de izquierdas. Luego seguirá a Buenos Aires donde vive, trabaja, publica sus primeros libros y tiene a sus hijos. De allí a Colombia donde reside unos años para volver a Argentina y desde allí, amenazado por la ignominia de las dictaduras, seguir en 1979 a España, donde vive ininterrumpidamente hasta su muerte, acaecida en noviembre de 2009 a los casi ochenta años de su edad. Preferentemente lo hace en Andalucía: Jaén, Torredonjimeno, Málaga pero también con estancias en Madrid, Alzira y aun otros lugares en un tránsito continuo que no se detuvo hasta el final.

Las implicaciones de este nomadeo, pese a ser un fenómeno que proviene directamente de su biografía, de su situación histórica, significa la obra poética al localizarla en un lugar problemático respecto a las interpretaciones que siguen siendo predominantes en nuestro contexto cultural. José Viñals fue consciente de la ambigua ubicación que le tocó en suerte e hizo de la voluntad, la solidaridad y el afecto los vínculos fundamentales con los lugares y los tiempos por los que transitó. Es decir, hizo de la nacionalidad más que un dato consabido el tipo de puzzle que la postcolonialidad nos ha enseñado a resolver. Teniendo muy presentes las identidades complejas surgidas a su amparo y su irreductibilidad a patrones preconcebidos de cultura o raza. En lo que profundiza Bhabha cuando, en The Location of Culture, se refiere "al quiebre de la identificación cultural tradicional con un sistema estable de referencias que ahondan en la imposibilidad lo puro" (Bhabha 1994)2.

Sus ideas nos asisten a comprender las implicaciones de la resistencia que la vida y la obra de Viñals oponen a su ubicación sin paliativos en un solo espacio. Y así, más que una síntesis nos hallamos en su obra ante una hibridación en el sentido que otorga al término Bhabha: una tensión en la que las diferencias conviven sin elimi-

\footnotetext{
2 La traducción es nuestra.
} 
narse. Este rasgo pone de relieve una identidad construida mediante la superposición de herencias y experiencias propias, en las que la contradicción no es un signo de precariedad, sino uno de los rasgos fundadores.

Esto guarda relación con la idea de que el exilio y lo extranjero generan una marca insoslayable en lo vital y en la escritura. Pero una marca que se resiste a generalizaciones sobre el tipo de impacto que esta experiencia tendrá sobre el proceso intelectual. Volvemos a Said cuando nos dice "aunque es una condición de hecho, el exilio es también para mis propósitos una condición metafórica [...] mi diagnóstico del intelectual exiliado deriva de una historia social y política de dislocación y migración [...] pero no se limita solo a ella" (Said 1996: 52-53). El propio Viñals nos daba cuenta de la complejidad de los resultados del proceso de desplazamiento sobre la escritura.

Al perder contacto con los orígenes, no has perdido solamente ciertos signos del lenguaje, del habla, has perdido, además, una flora, una fauna, una geografía. En España hay un pájaro muy bonito, la urraca, que es negra con manchas blancas. En Argentina está también la urraca pero es gris clarita, casi blanca con manchas negras, exactamente lo opuesto, como el negativo y el positivo de una fotografía. Al perder el contacto con el mundo originario pasan muchas cosas esenciales; tienes que buscar un nuevo aprendizaje, tienes que hacerte cargo de que aquí existe una oropéndola que no existía en tu geografía. Tienes que entrar en universos que son nuevos, y que son nacionales, y así se vuelve enriquecedor el proceso de extranjerización, no de otra manera. Pero debes hacerte cargo de todo eso, aprender el nombre de las plantas más inmediatas, saber que lo que aquí se llama buganvilla, en Argentina se llama Santa Rita, y que en Michaux también existe y se llama clemátide... Yo creo que todo esto enriquece el universo del poeta. (Viñals en Fisher y Del Pliego 2008: 328)

Bueno, tuve grandes problemas de adaptación, lo cual es fácilmente entendible, me parece a mí. De adaptación a la lengua y al habla de España en primer lugar. Fueron años de aprendizaje, de integración. A mi me costó algunos años dar con lo que yo llamo el genio de una lengua. Son bastante distintos el castellano que se habla en España y el castellano que se habla en Latinoamérica, los dos hemisferios de una misma esfera, como lo he dicho otras veces. Mi lengua natal era la materia plástica con la que yo trabajaba; sus vocablos y su sintaxis eran mis pomos de óleo y mi paleta a los que yo no encontraba equivalentes en el habla española. La necesaria armonización y la indispensable complementación llevó años en ocurrir, años no exentos de algún sufrimiento creativo cuando no de parálisis. (Viñals en Fisher y Del Pliego 2005: 90)

Estos matices también los encontramos mirando en sus poemas. Donde lo híbrido se abre paso para dar cabida a lo que no responde al modelo maniqueo de contraposiciones -nosotros/ellos, familiar/extraño-, ni puede reducirse al Et pluribus unum que tanto gusta a las culturas nacionales. Si, como hace en el poema "Espejo espejo" (incluido en Milagro a milagro) resumimos el debate al que su posición le enfrenta mediante la contraposición de dos adverbios de lugar ("aquí o allí"), la única solución posible es responder con una pregunta que deconstruya la lógica implícita de la oposición (“¿Dónde es aquí y dónde allí?”) y la eleve por encima de 
las barreras que impiden al lenguaje fijar su verbo en lo que no tiene ni lugar ni tiempo porque está siempre y en todas partes. En "Espejo espejo" la imposibilidad de contestar a ese dónde impertinente se desploma mediante una oblicua referencia a la muerte ("el agujero en tierra [...] al borde mismo [...] de mi descalzo de uñas frías, tenuemente moradas") que todo lo iguala. En "Así se inician siempre las grandes conversaciones" (incluido en Jaula para Juan), ante un dilema similar relacionado con su "exilio", extranjería o "trashumancia", el poema zanja el debate con un abrupto giro hacia la otra gran salida: "Dicho en pocas palabras -concluye el poema-: te amo" (Viñals 1995, 2: 199-281).

El vértigo que se manifiesta en los poemas recién mencionados es una muestra más del difícil acomodo que encuentra Viñals en las ideas preconcebidas. Su originalidad también se da en otro de los temas que cruzan su obra en relación con el exilio; nos referimos al viaje. El asunto es central porque nos ofrece una muestra de la independencia con que elige su postura. El del viaje es un tema ampliamente mitificado en la escritura moderna y el poeta se encuentra en una posición propicia para darse una capa de prestigio que, sin embargo, nunca se ha tratado de aplicar. Lo llamativo es aquí que la recreación del viaje encuentre en su obra una postura diferenciada de la del núcleo de autores vanguardistas con el que su poética y su predilección le conectan en muchos otros sentidos.

El viaje fue para la vanguardia uno de los asuntos que más vivamente excitaron su interés vital y literario. Desde el último de estos espacios se fraguó toda una mitología que identificó sin paliativos el viaje como una de las formas privilegiadas de conocimiento e independencia personal. El tiempo y la crítica nos han hecho ver lo que esto tiene de falaz y de arriesgado, matizando así alguno de sus excesos. La crítica contemporánea ha señalado la preponderancia de ciertas asociaciones automáticas entre desplazamiento y ciertas formas, presuntamente privilegiadas, de conocimiento y comprensión surgidas a partir del Romanticismo y que encuentran en las vanguardias internacionales una ratificación acrítica, aureolada del prestigio elitista que también presidió otros aspectos de su poética según, entre otros críticos, Renato Poggioli (1964).

Todavía hoy la literatura sigue favoreciendo una imagen homogénea de una situación histórica en la que, sin embargo, los desplazamientos son mucho más diversos y nos afectan de un modo cada vez más complejo. Karen Kaplan (1996) ha hecho un seguimiento de la forma en que estas ideas se perpetúan en la literatura ensayística. De acuerdo con su análisis, las vanguardias adoptaron la metáfora del exilio como una de las formas de distanciamiento político e histórico favorable a sus categorías estéticas. Esta estrategia, vinculada al ideal poético de extrañamiento y enajenación, sancionan el desplazamiento como una forma idónea para la observación del propio sujeto y un modo de alzarse sobre los condicionantes históricos para producir su obra. Mediante estas operaciones se incurre en una generalización que podría reducir todo tipo de viaje a una mera marca de prestigio. En este tipo de discursos, que identifican con demasiada facilidad escritura y expatriación, el viajero puede convertirse en un testigo aséptico, un turista de la vida de los otros, desactivando así el potencial transformador inscrito en este cambio de lugares y tradiciones. La acuñación de tópicos sobre el viaje puede derivar en una clausura de las pre- 
guntas que debería abrir y en la aceptación conformista de diferencias absolutas de los binomios casa/lejanía, centro/periferia. Estas afirmaciones nos recuerdan que es arriesgado hacer juicios a priori respecto al alcance y los logros de las obras de los que se presentan a sí mismos como desplazados.

La tradicional celebración de la aventura viajera o del exilio, la reducción del desplazamiento a la experiencia cosmopolita de minorías, son ideas que no se avienen con las alusiones al tema con las que el lector se encontrará en la obra de José Viñals. Casos como el suyo ejemplifican con mayor acierto la situación postmoderna tanto por el tipo de desplazamiento que reflejan, como por la manera en que se presenta su periplo.

Viñals está lejos de celebrar acríticamente los viajes. Como ya hemos visto, puede presentar el asunto bajo cierto halo de angustia o desestimarlo como la actividad menos provechosa de su vida -en "Nupcial" (aparecido en El amor)-. Por otra parte, su obra poética y narrativa explicita que el suyo no es un viaje de placer ni un exilio estético; aunque su llegada a España estuvo forzada por la situación política de su país natal, su filiación emocional y su disposición anímica le llevan a identificarse con un tipo de traslado mucho más común y menos prestigioso en nuestro siglo, la emigración. En contraposición directa con la postura de ciertos autores de vanguardia con quienes, por lo demás, Viñals se siente fuertemente emparentado, es especialmente interesante el poema "Un bárbaro en Asia", incluido en Transmutaciones. En él la voz poética desdoblada debate si debe o no seguir el ejemplo del famoso escritor (y viajero) belga, Henri Michaux. Aunque el poema es un elogio a Michaux y un menosprecio a la voz poética en que se proyecta el autor, el debate interno le lleva a cuestionar el papel de aventurero, apropiado para el belga pero no tanto para el autor; así concluye reivindicando el ejemplo creativo, pero apartándose del vital: "Pero no mires su sandalia - dice Viñals de la de Michaux- pues tu pie no se ajusta a su medida" (Viñals 2000: 39). No es que el autor rechace el potencial de conocimiento que puede estar ligado al viaje, sino que no se conforma con la asociación al uso que la tradición confiere a esta experiencia. Y este poema no es una excepción.

Otro singularmente significativo, "Conveniencia de instalar letreros de entrada y salida a cada lado de las puertas", incluido en 72 lecciones de ignorancia, vuelve a abordar de forma crítica la relación entre migración y cierto tipo de conciencia, muy estimada por las vanguardias y que con frecuencia se ha asociado al viaje: el sueño. El poema parte de una cita del poeta y ensayista vinculado con el simbolismo francés, Leon-Paul Fargue (1876-1947) -“Soñé tanto que ya no soy de aquí"- y la desmonta recurriendo al tipo de pensamiento, más poético que filosófico, que caracteriza buena parte de los libros escritos en Argentina y Colombia. El poema presenta una batería de objeciones lógicas que termina por evidenciar la simplificación de la asociación entre el sueño y la extranjería. El texto no despeja dudas, sino que crea un laberinto de ideas que ejemplifica el calado de las contradicciones implícitas en el enunciado de partida. El aspecto emocional desborda el analítico y pone en evidencia que el asunto de la migración, complejo y doloroso, no puede ser reducido a tópicos y banalidades.

José Viñals utiliza, en este y en otros poemas, la estrategia de dejar de lado la necesidad de optar; prefiere la suspensión de toda pregunta y desvía la mirada hacia 
un espacio donde es posible la afirmación de sus vivencias más allá y por encima de nostalgias y geografías, o subrayando la imposibilidad de restañar la brecha de dolor que abre la distancia. Este último es el caso que predomina en los poemas "Antónimos" de Telón de boca, "El padre" de Doble concierto para laúd y fémur, o "Espejo espejo", incluido en Milagro a milagro. En este último, especialmente elocuente, la tensión se encarna lingüísticamente en la contraposición de dos adverbios de lugar ("aquí o allí"); el enfrentamiento pone en evidencia el desgarro vital y da lugar al mismo tipo de reacción observada en "Conveniencia de instalar letreros de entrada y salida a cada lado de las puertas": la única respuesta es el cuestionamiento de la lógica implícita en la oposición, la negación de la validez de la lógica binaria que condensa: “¿Dónde es aquí y dónde allí?”; la voz poética, ligada sin distinción a los espacios que se tratan de diferenciar, no tiene forma de otorgar referentes diferenciados a estos adverbios, sencillamente elude el dilema y, al hacerlo, elude la trampa del reduccionismo.

Este gesto elusivo se aprecia también en el poema "Así se inician siempre la grandes conversaciones", incluido en el primero de los libros de la época colombiana, Jaula para Juan, pero aquí se escapa por una tangente afirmativa, ya que contrapone al dolor de la extranjería la celebración del amor. En este poema la mención de dos objetos que el autor vincula metafóricamente con el desplazamiento -unos zapatos que llevaba puestos "la primera vez en mi vida que me sentí extranjero" y un reloj que "estuvo en todos los sitios conspicuos que no puede desconocer ningún viajero que se precie" - terminan revelándose como una excusa para algo, al parecer, mucho más importante, una declaración de amor. Zapatos y reloj le facilitan

[...] unos pocos minutos y algunas anécdotas itinerantes como pretexto para eludir tus ojos y el musgo de tus ojos y la tierra de tus ojos y la inmovilidad de tus ojos observando sin entusiasmo mis ingenuas mentiras,

la verdad con la que trafico sin darle demasiada importancia ni a mis zapatos ni a mi reloj. (Viñals 1995, 2: 199-281)

La lectura de estos poemas podría darnos a entender que el autor rehúsa asociarse con el aventurero, trata de desarticular la identificación automática entre viaje y revelación y pone en entredicho los fundamentos lógicos de la reflexión sobre el desplazamiento; sin embargo, no por ello deberíamos precipitarnos a concluir que todo lo relacionado con el desplazamiento implica una consideración negativa. Hay al menos un lugar, connotado de forma muy favorable, a la que el recuerdo de sus traslados y mudanzas le lleva de manera inequívoca: la solidaridad. Su peregrinaje crea lazos con los otros, extiende mucho más allá de los lazos sanguíneos su familia. Viñals plantea la migración como una actividad grupal y solidaria, no individual y escapista. Los personajes de los poemas "Aquellos andenes" y "Nupcial" son parejas, no individuos, y hablan en plural de su itinerancia. Esto compensa, en parte al menos, las desdichas de las separaciones y, en cuanto estas pasan a ser parte de una historia compartida con los que viajan junto al poeta, llegan a tener connotaciones más optimistas, como ocurre por ejemplo en el poema que da título a Cuan larga eres. En otras ocasiones, como en el poema "Conjunción de los métodos dialéctico 
y psicoanalítico", de 72 lecciones de ignorancia o en el largo poema narrativo El Osar, incluido en el volumen He amado, la voz poética desarrolla la historia de una emigración familiar en la que el autor es sólo uno de los participantes en la experiencia de sucesivos arraigos y desarraigos. Solidaridad con el clan, y solidaridad también con los desconocidos que va encontrando. Los lugares que han formado parte de ese itinerario no son solo nombres en la larga lista de ciudades visitadas a mayor gloria del distanciamiento estético y de uno mismo. El autor subraya la importancia del entramado fraternal de relaciones establecidas. "Un violín y los hechos", de Telón de boca y "Bogotá", incluido en Animales, amores, pasajes y blasfemias son ejemplos especialmente claros de esta certidumbre.

La hermandad se teje y desteje al mismo tiempo que rememora su migración. Conciencia de clase social, de comunidad, de familia, que se mezcla en la escritura con su ese otro linaje de "sangre que ahora es tinta" -como ha dicho Jason Wilson (1995: 26)-, la tradición a la que asocia sus poemas. Esta solidaridad es tan importante en su obra que no sorprende que el viaje de Viñals nos conduzca hasta ella. Solidaridad, otra de las columnas que sostienen su obra, otro de los nudos para enlazar poesía y política.

El otro aspecto del exilio de José Viñals es el exilio interno. Algo que podría ser paradójico para quien viene desde lejos, desplazándose. Pero no lo es debido al lugar en que su práctica poética sitúa a Viñals en el escenario de la poesía española. Cuyos ocupantes, sin desplazarse, experimentaban la situación metafórica del exilio en su propia tierra. Uno de sus poemas fuertes, "Dinastías" (incluido en Alcoholes y otras sustancias), nos da pistas de su situación y sus alianzas.

Los nervios pesan y Artaud, el desmesurado, lo sabía. Hay cabellos de dios en el prostíbulo y Leautréamont lo sabe.

Hubo berlinas detenidas en lo secreto de la noche, y Milosz, ya en su tiempo, lo contaba.

Hubo gallinas en los cementerios, cuervos en los trigales, minotauros, leprosos, parturientas, ahorcados en la Torre de Nesle, enanos góticos como Scarbó, y un misógino loco en el castillo de Braganza. Hubo, al menos, tres estupendos alucinados; uno era un Rey; otro, Alonso Quijano y, el tercero, un marqués sifilítico que estudió los rebrotes de la crueldad.

Y luego vengo yo, que como gallina en pepitoria y eructo sin piedad contra el rostro pulido de la luna. (Viñals 1995, 3: 543-620)

Desde la postguerra la crítica oficial, la mediática y la académica, la que fija el canon en España, ha legitimado un tipo de poesía basada en los aspectos más estáticos de la tradición, anclados en la representación y en un uso normalizado del lenguaje. Esto se hace patente, quizá de forma paradójica en la Transición, período histórico que redefine, complejizando la identidad social, política y cultural española hacia la diversidad, contestando el absolutismo uniformador y opresivo prevalente no solo en la dictadura franquista sino desde antes.

En poesía, sin embargo y de acuerdo con Guillermo Fernández Rojano (1998: 9), se producía ese pacto de normalización que insistía en la operación de formar un canon vinculado a propuestas hostiles hacia los lenguajes artísticos de la 
(post)modernidad. En el caso muy concreto de comienzos de los ochenta, aquel que legitimaba a la tendencia realista, conservadora en el uso del lenguaje y anclada en la mímesis y la representación, que además formó una tendencia (dominante) que hegemonizó el escenario sancionando para las poéticas que se escapaban de él, en palabras de Marcos Canteli (2008), una situación de "invisibilidad" o, al menos, de "visibilidad borrosa", de esa "intermitencia entre la aparición y la desaparición" a la que se refiere el "parpadeo" que da título a su estudio.

La poesía de Viñals es una que se aleja ampliamente de este discurso normalizador que opera de una forma reduccionista con las potencialidades de la poesía. Y es que para Viñals la poesía es exploración y aventura en lenguaje, haciendo suyas las concepciones que Jakobson hace para la poesía en cuanto a una reevaluación radical del discurso con la consecuente particularidad de su resultado. Eso que lleva a la poesía, en palabras de Miguel Casado a constituir un "estado de la lengua". A una problematización del lenguaje que no elude situaciones de conflicto y transgresión.

Así, no es de extrañar que el linaje de autores al que Viñals se vincula sea justamente el de los que transitan por estos derroteros. Gamoneda entre ellos, cuyas concepciones del poema como objeto autónomo, de la poesía como generadora de una realidad en sí, engarzan notablemente con su escritura. Con aquellos cuya noción de compromiso es amplia, alejada, siguiendo a Méndez Rubio (2008), de lo puramente realista, voluntarista, inercial o incluso autoritario.

Estas características y estos vínculos ubican a Viñals de lleno en los discursos críticos. Con ellos, genera una poesía exigente, rica en elementos que operan como capas de sentido renunciando a toda concepción unitaria del poema y que, en contra de lo asumido por la crítica conservadora, no renuncia a una inserción plena en el mundo. Inserción amplia en elementos estéticos pero que de ninguna manera renuncia a lo ético y sus aledaños sociales o ideológicos, asuntos clave en la poesía de Viñals pero tratados desde la totalidad lingüística y referencial que la compone. Poesía que, como el mismo poeta expresa, es una "actividad del espíritu", entendido éste en un sentido "laico y materialista como totalidad que concentra todas las energías humanas: corporales, genitales, fisiológicas, mentales, psicológicas, genéticas, gregarias, individuales, sociales" (Viñals 2003: 26).

Esta aproximación es en parte responsable de que la obra viñaliana tarde en encontrar sus cauces de circulación en España. Aunque esto de ninguna manera hace que la producción de Viñals se detenga, pues continúa siendo en este país el escritor prolífico que venía siendo en Latinoamérica. Sin embargo, quince años transcurren antes de que se publique su influyente Poesía Reunida en tres volúmenes, en 1995 y que contiene poesía escrita en Argentina, Colombia y España. Que justamente comienza a circular entre quienes participan de los discursos críticos, aquellos sometidos al proceso de invisibilidad al que nos referíamos. Que no impiden que desde la subsecuente publicación de Animales, amores, parajes y blasfemias en 1998, la obra de Viñals comience a hacerse muy visible y a publicarse asiduamente a lo largo de la década venidera, provocando así esa intermitencia en el ver propia de quien (para sacar de sí algo que irrita o para ver mejor lo sorprendente) parpadea. 
En la afiliación de Viñals con este sector de la poesía española tiene un lugar central el linaje vanguardista asumido y manifestado por el autor en sus ensayos. Pero este vínculo actúa de acuerdo con la época que le toca, ésta, en que las vanguardias ya no existen como movimientos organizados, lo que es un dato clave para entender la vigencia de su legado. Este es un hecho interesadamente desconocido por la crítica conservadora, que se refiere a ellas de una forma rígida, despectiva y hasta caricaturesca, como si siguieran existiendo a la manera de los años 20 y 30 . Ignorando que la transformación experimentada desde la segunda postguerra, con la disolución del elemento militante, implica formas diferentes de operar y de existir que matizan sin atenuar su gravitación en el arte (post)moderno.

La aproximación de Viñals hacia las vanguardias es heterodoxa y no incluye elementos militantes o miméticos respecto a los grupos organizados, ya muy atenuados o inexistentes en sus años formativos. Actitud en la que profundizamos con las palabras de Molina Damiani cuando nos habla de la heterodoxia de la aproximación vanguardista de Viñals y sus referentes, tanto en ellas como en sus precursores. Así, imbricados, encontramos "los hallazgos del modernismo feísta, de las lecciones del romanticismo maldito, de las direcciones del simbolismo visionario, del surrealismo objetivo que vivifica el alcohol de los sueños, los retos del realismo que no renuncia a atender lo irracional" (Molina Damiani 2007: 19).

Cobran importancia aquí las reflexiones de Octavio Paz en cuanto a las vanguardias como el fin de la "tradición de la ruptura" en la poesía moderna originada en el Romanticismo. Las vanguardias históricas y su actuación radical suponen la definitiva atomización de la tradición, que se fragmenta tras la gran ruptura vanguardista y ya no constituye un referente monolítico contra el cual rebelarse. Paso clave hacia las epistemologías (post)modernas con sus nociones relativas de lo verdadero y lo bello. Que no niegan la validez del legado de las vanguardias sino que atenúan el alcance de sus pretensiones maximalistas y redentoras en cuanto a su actuación como gran relato.

Así, el poeta hace suyos elementos clave del ethos vanguardista y los adapta a su época y su andadura. Integración arte/vida, crítica a la representación, la poesía como fundadora de una realidad autónoma existente en el lenguaje, a través del que se genera una actitud de exploración y aventura. De crítica, conflicto y cambio. Donde tienen un lugar importante los elementos constructivos y formales de la lengua, que Viñals trabaja en una dirección muy particular llegando a la constitución de un sistema expresivo.

La vanguardia ocupa un lugar central en la tradición poética hispanoamericana, de cuya fundación y desarrollo es un elemento inalienable. Esta presencia en la tradición de la poesía española también es amplia aunque más problemática, debido a los intentos, según Méndez Rubio (2008), de la crítica conservadora de minimizar su impacto. Se hace necesaria entonces, para situar adecuadamente la poesía de Viñals, una concepción de la vanguardia entendida, siguiendo a Miguel Casado, como un nuevo concepto contemporáneo de tradición y no desde una forma esclerotizada y rígida, anclada en la forma de actuación de las vanguardias históricas.

Así, desde su formación en Argentina, Viñals tiende puentes en este sentido. Ya hemos mencionado como en la elaboración de su paideuma son claves los precur- 
sores del surrealismo, luego algunos de sus miembros y posteriormente sus epígonos latinoamericanos del Cono Sur. Construcción crítica de su propia tradición que se extiende a otros poetas de esta órbita que serán clave en su desarrollo como Vallejo o Huidobro. Por eso no extraña que sus interlocutores en España sean poetas en los que justamente se puede rastrear el linaje vanguardista, que es parte importante del sustrato que ejerce de malla entre quienes cultivan las poéticas críticas.

(Est)ética vanguardista que lleva a Viñals hacia una práctica poética transgresora, que cuestiona y amplía los límites de lo poético. Que agita el lenguaje haciéndolo, con Gamoneda, "que diga cosas que de otro modo no serían dichas". Que asume, con Bretón, la insuficiencia de considerar a la poesía como un "género literario". Con Paz, la amplitud de lo poético desde "lo erótico a lo político; de lo lingüístico a lo revolucionario".

Hace poco más de un año Viñals marcho al exilio definitivo, la muerte. Estas son las últimas estrofas del poema que cerraba el libro postrero publicado por el poeta en vida, El Silencio y las Grietas. Ya los hay póstumos. En él, a su manera, da cuenta del viaje definitivo, del exilio radical que se le acerca.

Aquel que va a morir no tiene ya memoria del amor, de los años. Aquel que va a morir tiene una lenta prisa. Tiene morados ya los dedos de los pies y las manos. Una tela de araña atenúa la luna del espejo. Duermen los libros en los cautos estantes. Hay un polvillo ceniciento en los muebles y enseres. Gotea la escarcha en la techumbre. Ha caído una mosca en el agua del vaso.

Alta es la noche, indecisa e informe la precoz madrugada. Ha cesado la música. Duermen las ratoncillos. En el establo el caballo medita. Alto el silencio, alto el fulgor del cielo, alta la madrugada. Breve el sigilo de la muerte. (Viñals 2006)

\section{BIBLIOGRAFÍA}

BHABHA, Hommi K. (1994): The Location of Culture. London: Routledge.

CANTELI, Marcos (2008): Transitar el parpadeo: seis poetas españoles. Durham: Duke University.

FERNÁNDEZ ROJANO, Guillermo (1998): “Prólogo”, en Viñals, José, Animales, amores, parajes y blasfemias, pp. 9-14. Valencia: Germanía.

FISHER, Andrés; y DEL PLIEGO, Benito (2005): "Es una cosa muy grande la poesía. Entrevista con José Viñals”. Hispanic Poetry Review, vol. 7, núm. 2, pp. 87-101.

- (2008): "Entrevista con el pájaro. Una conversación con José Viñals". Anales de Literatura Hispanoamericana, vol. 37, pp. 323-330.

KAPLAN, Karen (1996): Questions of travel. Postmodern Discourses of Displacement. Durham, Carolina del Norte: Duke University Press.

MÉNDEZ RUBIO, Antonio (2008): La destrucción de la forma (y otros escritos sobre poesía y conflicto). Madrid: Biblioteca Nueva.

MOLINA DAMIANI, Juan Manuel (2007): “Desde Jaén, donde reside". Lunas Rojas, núm. 15, noviembre de 2007. Monográfico dedicado a José Viñals, pp. 13-19.

POGGIOLI, Renato (1964): Teoría del arte de vanguardia. Traducción de Rosa Chacel. Madrid: Revista de Occidente. 
SAID, Edward (1996): Representations of the intellectual. Nueva York: Vintage.

VIÑALS, José (1995): Poesía reunida (1963-1993), 3 vols. Jaén: Ayuntamiento de Jaén.

—: 72 lecciones de ignorancia, en Poesía reunida, vol. 2, pp. 283-396. Jaén: Ayuntamiento de Jaén.

—: Alcoholes y otras substancias, en Poesía reunida, vol. 3, pp. 543- 620. Jaén: Ayuntamiento de Jaén.

—: Doble concierto para laúd y fémur, en Poesía reunida, vol. 3, pp. 470-541. Jaén: Ayuntamiento de Jaén.

—: Jaula para Juan, en Poesía reunida, vol. 2, pp. 199-281. Jaén: Ayuntamiento de Jaén.

—: Telón de boca, en Poesía reunida, vol. 3, pp. 419-470. Jaén: Ayuntamiento de Jaén.

- (1998): Animales, amores, parajes y blasfemias. Valencia: Germanía.

- (1999): Milagro a milagro. Madrid: Hiperión.

- (2000): Transmutaciones. Madrid: Visor.

- (2002a): El amor. Madrid: Hiperión.

- (2002b): Elogio de la miniatura. Barcelona: La poesía, señor hidalgo.

- (2003): "Habla". El Cielo de Salamanca. Revista cultural Euroamericana, núm. 44, pp. 25-30.

- (2005): He amado. Barcelona: La poesía, señor hidalgo.

-: Cuan larga eres, en He amado, pp. 213-260. Barcelona: La poesía, señor hidalgo.

-: El Osar, en He amado, pp. 533-562. Barcelona: La poesía, señor hidalgo.

- (2006): El silencio y las grietas. Santa Cruz de Tenerife: Idea.

WILSON, Jasón (1995): “Acerca de la poesía reunida de José Viñals”, en Viñals, José, Poesía reunida (1963-1993), 3 vols, pp. 13-29. Jaén: Ayuntamiento de Jaén. 